in vivo $34: 3187-3193(2020)$

doi:10.21873/invivo.12154

\title{
A Novel Lipofuscin-detecting Marker of Senescence Relates With Hypoxia, Dysregulated Autophagy and With Poor Prognosis in Non-small-cell-lung Cancer
}

\author{
ALEXANDRA GIATROMANOLAKI ${ }^{1}$, MARIA KOUROUPI ${ }^{1}$, \\ KONSTANTINA BALASKA ${ }^{1}$ and MICHAEL I. KOUKOURAKIS ${ }^{2}$ \\ Departments of ${ }^{1}$ Pathology, and ${ }^{2}$ Radiotherapy/Oncology, Medical School, \\ Democritus University of Thrace, Alexandroupolis, Greece
}

\begin{abstract}
Background/Aim: The role of senescence in defining tumor aggressiveness at a clinical level remains obscure. A novel mixed histochemical/immunohistochemical method (SenTraGor ${ }^{T M}$, STG) detecting lipofuscin accumulation allows the assessment of senescent cells in paraffin-embedded tissue material. Materials and Methods: STG expression was quantified in 98 surgically resected primary non-small-cell-lung carcinomas (NSCLC). Data were analyzed in parallel with other immunohistochemical markers related to hypoxia and autophagy. Results: Strong STG staining was noted in 36/98 cases (36.7\%). High STG expression was significantly associated with high HIF $1 \alpha$ expression and high expression of glucose (GLUT1) and monocarboxylate (MCT2) transporters, pointing to a link between senescence, hypoxia and glycolysis. High STG expression was also linked with high cytoplasmic accumulation of MAPI-LC3B, TFEB and LAMP2a, suggestive of a blockage of autophagy flux in tumors with intense senescence. Survival analysis showed a direct association with poor survival, independently of stage. Conclusion: SenTraGor ${ }^{T M}$ provides a reliable methodology to detect lipofuscin accumulation in cancer cells in paraffinembedded tissues, opening a new field for translational studies focused on senescence.
\end{abstract}

This article is freely accessible online.

Correspondence to: Alexandra Giatromanolaki, MD, Department of Pathology, Medical School, Democritus University of Thrace, Alexandroupolis, Greece. Tel: +30 2551352117, e-mail: agiatrom@med.duth.gr

Key Words: Senescence, lipofuscin, lung cancer, hypoxia, glycolysis, autophagy.
Following exposure of cells to various external damaging stimuli, cells may enter the status of a permanent cell cycle arrest. During the life of an organism, tissues are enriched by senescent cells creating a pro-inflammatory microenvironment that facilitates the development of degenerative diseases or tumorigenesis (1). Nevertheless, entrance of damaged cells in senescence may also have protective effects, as cell cycle arrest prevents cells with mutations to progress to cancer development (2). Senescent cells are not inactive cells and, in contrast, they exert potent bystander effects, also contributing to the development of the so-called senescence-associated secretory phenotype (SASP), interfering with tissue repair and remodeling processes or even modulating local immunity (3-5).

In the absence of reliable and specific methods for the identification of senescent cells in paraffin-embedded tissue sections, senescence as a tumor-related phenomenon and its role in defining local and metastatic aggressiveness at a clinical level remains unclear. Despite the well-established effect of radiotherapy and cytotoxic therapy in inducing senescence (6), the lack of relevant biomarkers that work at the tissue level has prevented the study of senescence as a tumor and normal tissue feature that regulates response to therapy and treatment toxicities $(7,8)$.

Many biological processes characterizing senescent cells have become targets for the development of biomarkers to quantify senescent cells in tissues. Among them is the accumulation of organelles (lysosomes, endoplasmic reticulum and mitochondria), changes in the composition of nuclear and plasma membrane, chronic DNA damage and secretion of proinflammatory factors and molecules related to tissue remodeling (9). The activity of the lysosomal senescence-associated betagalactosidase (SA-bgal) is widely used to detect senescent cells in live or frozen cells and tissues (10). Application of such a method is not feasible in paraffin-embedded tissues. An alternative histochemical method is the Sudan Black B (SBB), where the reagent selectively binds to lipofuscins and labels 
accumulating dysfunctional lysosomes (11). Recently, Gorgoulis et al. developed a sensitive mixed histochemical/ immunohistochemical method (SenTraGor ${ }^{\circledR}$, STG) that detects senescent cells. This is based on the synthesis of a hapten-linked SBB analog (12). This method allows the assessment of senescence in paraffin-embedded tissue material (13).

In the current study, we validated the STG method in a series of non-small-cell-lung carcinomas (NSCLC). We show that tumors with increased number of cancer cells expressing lipofuscins, which eventually become senescent cells, are clinically aggressive and characterized by significant hypoxia and dysregulated autophagy pathways.

\section{Materials and Methods}

Materials. Tissue material from 98 surgically resected primary NSCLC were analyzed. These were serial cases according to the day of surgery. Patients who received radiotherapy or chemotherapy before or after surgery were excluded from the study. The age of patients ranged from 32 to 81 (median age: 68). Twelve of the 98 patients were female. Forty-six of the patients had stage I, 22 had stage II and 30 had stage III disease (Union for International Cancer Control system). Fifty-eight of cases were squamous-cell carcinomas, 22 were adenocarcinomas and 18 were undifferentiated large-cell carcinomas. The median follow-up of patients was 46 months, ranging from 26 months to 112 months.

Ethics. The Hospital Scientific Committee and the Ethics Research Committee of the Democritus University of Thrace and University General Hospital of Alexandroupolis approved the study (approval number ES11-26-11-18). The study was conducted according to the criteria set by the declaration of Helsinki. The study is retrospective on 'existing holdings' and informed consent was waved by the Ethics Committee, as analysis is performed anonymously on archival material (Human Tissue Authority, E Research, Code of Practice and standards (14).

Lipofuscin (SenTraGor ${ }^{T M}$ ) immunostaining. Three $\mu \mathrm{m}$ tissue sections from formalin-fixed paraffin-embedded (FFPE) material were cut and mounted on positively charged glass slides. SenTraGor ${ }^{\mathrm{TM}}$ (STG) (Cat no: AR8850040, Arriani pharmaceuticals, Attika, Greece) reagent was used to stain lipofuscin granules in cancer cells. Details of the mixed histochemical/immunohistochemical method applied have been previously reported by our group (13). The percentage of tumor cells expressing STG in the cytoplasm was assessed in all-optical $\times 200$ fields. The mean score was used to score each case.

Other immunohistochemical studies. Hypoxia and aerobic/anaerobic metabolism of cancer cells were assessed using immunohistochemistry for multiple enzymes/proteins. These data were available from a previously published study, where details on the antibodies and methodology are reported (15). Detection of glucose transporter proteins GLUT1 and GLUT2 was applied to assess glucose absorption. For the assessment of lactate kinetics we detected the monocarboxylate transporter MCT1 and MCT2 expression. Hexokinase II (HXKII), phosphofructokinase 1 (PFK1), and aldolase were detected to assess pyruvate transformation to glucose. The anaerobic usage of pyruvate was assessed by examining the expression of lactate dehydrogenase isoenzyme LDH5 (composed by $4 \mathrm{M}$ subunits). To assess the aerobic usage of pyruvate we assessed the expression of pyruvate dehydrogenase (PDH). Its phosphorylated inactive form (pPDH) and the PDH-kinase PDK1 (that phosphorylates/inactivates PDH) were also assessed. Enzymes of the Krebs cycle, like citrate synthase CSynth and isocitrate dehydrogenase IDH, were also assessed. We also detected the expression of Glucose6-phosphate dehydrogenase (G6PD), a key enzyme in the pentose phosphate pathway. The hypoxia inducible factor $\mathrm{HIF} 1 \alpha$ was detected using the ESEE122 mouse monoclonal antibody (University of Oxford, Oxford, UK).

Data from studies on autophagy and lysosome-related markers were also available and details on antibodies and methodology have been reported in relevant studies $(16,17)$. Briefly, The MAP1-LC3A and LC $3 \mathrm{~B}$ were used as targets to detect autophagosome membrane proteins. The Beclin-1 autophagy signalling molecule was also examined. The LAMP2a lysosome membrane protein and Cathepsin-D, an intra-lysosomal enzyme, were assessed. Moreover, the transcription factor TFEB regulating lysosomal biogenesis was also studied.

Ki-67 (Mouse monoclonal antibody, clone MIB-1, ImmunoLogic) was used to assess the proliferation index. Moreover, we assessed the expression of two markers involved in senescence, namely p16 (Rabbit clonal antibody, clone R19-D, Biotech) and p21 (Mouse monoclonal antibody, clone SX118, DAKO).

Statistical analysis. Statistical analysis was performed using the GraphPad Prism 7.0 package and the SPSS (v14.0, SPSS Inc.) program. To compare between categorical variables or grouped continuous variables, we applied the Fisher's exact $t$-test or the unpaired two-tailed $t$-test, as appropriate. Linear regression analysis was applied to assess correlations between continuous variables. The Kaplan-Meier method was used to assess the impact of the immunohistochemical variables on the overall survival of patients. Multivariate analysis was applied by a Cox proportional hazard model assessing the effect of variables on death events. A $p$-value of $<0.05$ was used for significance.

\section{Results}

Expression patterns. Three different patterns of SenTraGor ${ }^{\mathrm{TM}}$ (STG) expression were noted (Figure 1): Lack of expression or sporadic expression in less than $10 \%$ of cancer cells (negative pattern), weak staining in 10-100\% of cancer cells (weak expression pattern) and strong staining in 10-100\% of cancer cells (strong expression pattern).

Lack of expression was noted in 27/98 cases (27.6\%) and weak expression in 35/98 cases (35.7\%). Strong expression was noted in $36 / 98$ cases $(36.7 \%)$. Tumors with lack or weak expression were considered to have 'low STG expression', while tumors with strong expression in $10-100 \%$ of cancer cells were considered to have 'high STG expression'.

The distribution of the percentage of cancer cells with strong expression in our series of tumors is shown in Figure 2a. The percentage of cancer cells with strong STR expression ranged from $0-90 \%$ (median $0,75^{\text {th }}$ percentile $50 \%$ ). In tumors with high STR expression, this percentage ranged from $10-90 \%$ (median $50 \%, 75^{\text {th }}$ percentile $70 \%$ ). 

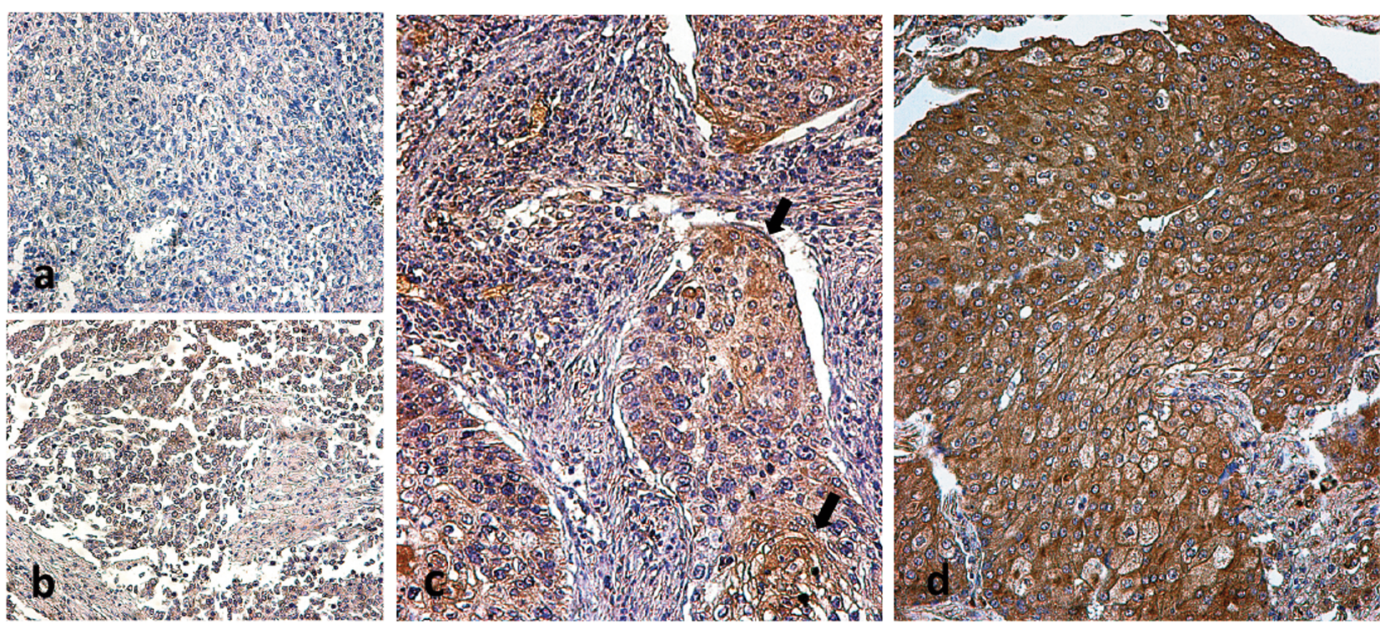

Figure 1. Immunohistochemical images of typical STG expression patterns: (a) lack of expression, $(b)$ weak expression, (c) strong expression in a specimen scored as having 30\% of STG-positive cancer cells (arrows) and, (d) strong expression in a specimen scored as having $90 \%$ of STGpositive cancer cells.

Association with histopathological variables. High STG expression was more frequently recorded in early-stage tumors (stage I) compared to more advanced stage II/III cases, but the difference did not reach significance (30/46 vs. $25 / 52$ cases; $p=0.18)$. No association with histology type or histological differentiation was noted $(p>0.67)$.

Association with hypoxia and metabolism proteins. High STG expression was significantly associated with high HIF1 $\alpha$ expression (mean \pm SD: $30 \pm 35$ vs. $49 \pm 33 ; p=0.01$ ). Among the rest of metabolism-related proteins examined, high STG expression was directly linked with high \% of MCT2 (mean \pm SD: $8 \pm 18$ vs. $19 \pm 23 ; p=0.005)$ and GLUT1 (mean \pm SD: $28 \pm 29$ vs. $40 \pm 34 ; p=0.05$ ) expressing cancer cells (Figure 2b). This was also confirmed in linear regression analysis $(p=0.006, \mathrm{r}=0.26 ; p=0.02, \mathrm{r}=0.30 ; p=0.05, \mathrm{r}=0.18$; for HIF1 $\alpha$, MCT2 and GLUT2, respectively).

Association with autophagy proteins. In linear regression analysis, high STG expression was significantly associated with high cytoplasmic expression of the autophagosomal marker LC3B and with a high cytoplasmic expression of the lysosomal biogenesis transcription factor TFEB $(p=0.005 / \mathrm{r}=0.28$ and $p=0.03 / \mathrm{r}=0.23$, respectively, Figure $2 \mathrm{c}$ and $\mathrm{d}$ ). Nuclear expression of TFEB did not, however, correlate with STR expression. Analysis in the group of tumors with high STG expression showed that STG expression corelated with the expression of lysosomal marker LAMP2a $(p=0.01, \mathrm{r}=0.38$; Figure 2e), LC3B ( $p=0.01, \mathrm{r}=0.36)$ and TFEB $(0.04, \mathrm{r}=0.31)$.

Association with proliferation and other senescence markers. Tumors with high expression of STG had a significantly lower proliferation index (mean percentage of cells with Ki67 nuclear expression was $15 \pm 11$ vs. $36 \pm 29$; $p=0.002$ ). STG score was not related to p16 expression. A direct association of STG score with p21 expression was noted (mean percentage of cells with p21 cytoplasmic expression was $14 \pm 16 v s .28 \pm 27 ; p=0.03$, in tumors with low and high STG expression, respectively).

Survival analysis. Kaplan-Meier survival curves showed a direct association between high STG expression and poor postoperative prognosis $(p=0.0008$, hazard ratio $\mathrm{HR}=2.5)$. Stratified analysis in stage I and stage II/III patients showed again the same statistically significant negative prognostic value of STG expression $(p=0.01 / \mathrm{HR}=4.9$ and $p=0.03 / \mathrm{HR}=2.09$, respectively) (Figure 3).

In a bivariate model of Cox-regression analysis, including stage and STG status, both parameters were independently linked with death events $(p=0.0001, \mathrm{HR}=1.9$ and $p=0.003$, $\mathrm{HR}=2.4$, respectively).

\section{Discussion}

Accumulation of senescent cells in tissues is a hallmark of aging. Senescent cells negatively regulate tissue homeostasis leading to degenerative diseases and cancer (3). Senescence is also induced in cancer tissues for various reasons, e.g. chronic DNA-damage, oxidative stress, mitochondrial dysfunction, cytotoxic therapy, or oncogene dysregulation. A major obstacle in studying tumor senescence in translational studies is the lack of reliable biomarkers. All phenomena characterizing senescent cells, like DNA strand breaks, cell cycle arrest related to cyclin-dependent kinase inhibitors 
a

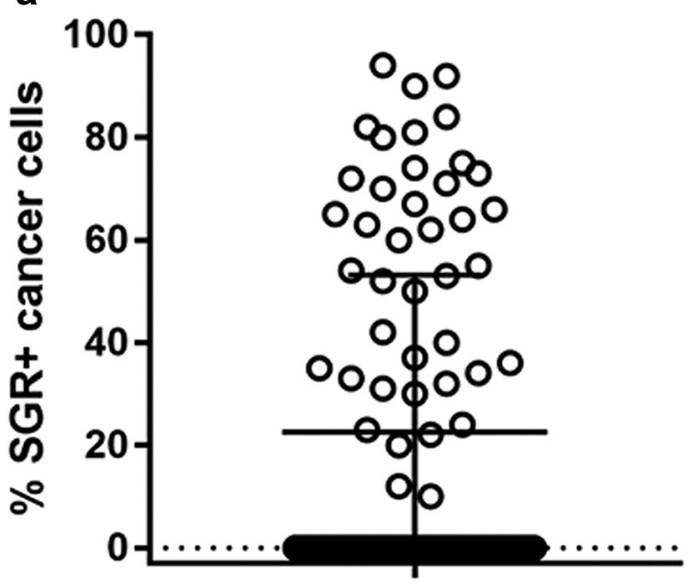

$98 \mathrm{pts}$
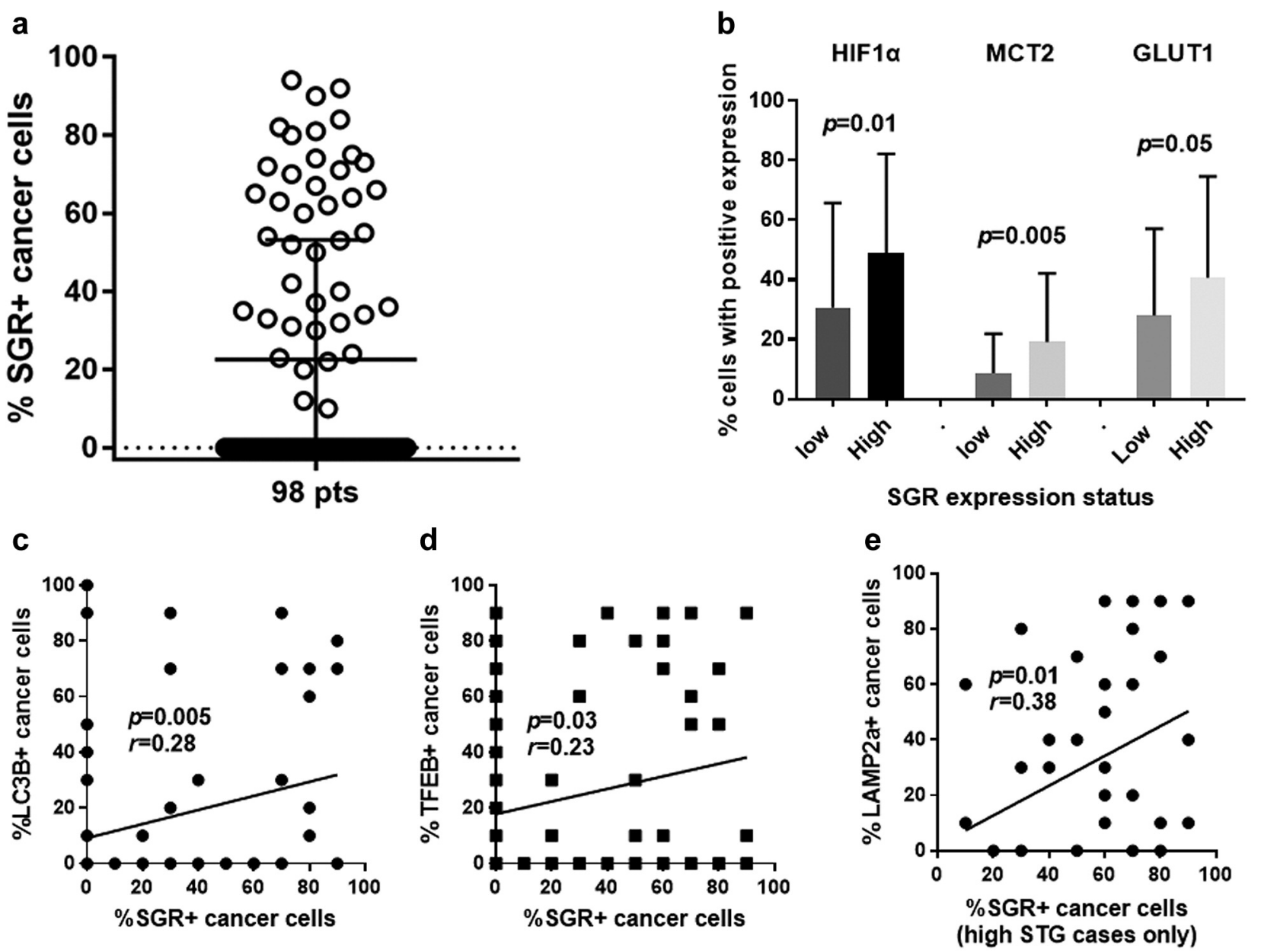

Figure 2. Statistical analysis figures: (a) Distribution of the percentage of cancer cells expressing strongly STG among 98 cases; (b) Percentage of cancer cells expressing HIF1 $\alpha$, MCT2 and GLUT1 according to the expression status of STG; (c) Linear regression analysis of the percentage of cancer cells expressing STG vs. the percentage of cancer cells expressing LC3B; (d) Linear regression analysis of the percentage of cancer cells expressing STG vs. the percentage of cancer cells expressing TFEB; (e) Linear regression analysis of the percentage of cancer cells expressing STG vs. the percentage of cancer cells expressing LAMP2a, in cases with high STG expression.

(like p16 and p21), expression of anti-apoptotic proteins (like the ones of the bcl-2 family), proteins involved in endoplasmic reticulum stress, accumulation of lysosomes or of mitochondria and others, are also features of biological processes independent of senescence (9).

The accumulation of lipofuscin or 'age pigment', is one of the strongest hallmarks of aging cells. Lipofuscins are polymeric plastic-like non-degradable materials, products of oxidatively modified proteins and lipid degradation residues, that accumulate into lysosomes $(18,19)$. Such lysosomes are not simply inactive, but in contrast, as they constantly receive new lysosomal enzymes in an attempt to get rid of this non-degradable material, they become a 'sink' depriving cells of valuable lysosomal enzymes. In this way, the physiological function of autophagy is impeded, blocking the cleansing of the cell from waste material. The only way for cells to reduce the lipofuscin content is through proliferation, as the initial content is diluted into two cells. Senescent cells, being unable to proliferate, continuously accumulate dysfunctional lysosomes containing lipofuscins.

In hematoxylin-eosin tissue sections, lipofuscin is detectable by optical microscopy. In 2006, Mahmoodi et al. published a study on prostate cancer patients, showing that detection of lipofuscin particles in prostate cancer cells was linked with low proliferation, low Gleason score and early clinical stage, suggesting that lipofuscin may be a marker of indolent disease (20). The recently developed histochemical/ immunohistochemical method SenTraGor ${ }^{\mathrm{TM}}(11,12)$ allows a far more reliable quantification of lipofuscin accumulation in paraffin-embedded tumor material, opening the road for 
translational studies focusing on senescence. The current, is the first-ever translational study examining the prognostic role of lipofuscin accumulation in human cancer, and more specifically, in lung cancer. High STG expression in tumors was directly linked with low proliferation index, compatible with a high percentage of senescent cells. STG was also related with another marker of senescent cells, namely p21, but there was no association with p16 expression. p21 and p16 proteins, although involved in senescence, are also involved in other biological pathways and are not considered reliable markers of senescence.

We further assessed the association of STG expression with hypoxia and metabolism markers. An immature vascular network or poor angiogenesis characterizing tumors is a common cause of intratumoral hypoxia leading to activation of the Hypoxia Inducible Factor HIF pathway (21). HIF1 $\alpha$ has been well recognized as a transcription factor characterizing senescence (22). During senescence, HIF1 $\alpha$ is upregulated and negatively regulates PGC1 transcription and expression (23). As PGC1 is a key factor regulating mitochondrial biogenesis (24), cellular energetics are deteriorated, resulting in the accumulation of ROS and in increased lipid oxidation, contributing eventually to the appearance of the senescence-associated secretory phenotype that interferes with tissue homeostasis and cell signaling (25). Hypoxia, therefore, eventually via HIF, promotes senescence and a glycolytic phenotype that explains the associations found in the current study between STG, HIF1 $\alpha$, glucose and monocarboxylate transporters.

Lysosomal accumulation is another feature characterizing senescent cells (26). The association between high STG and lysosomal membrane markers, like LAMP2a and TFEB, found in the current study, confirms the accumulation of lysosomes in STG expressing cancer cells. Lysosomal biogenesis is transcriptionally regulated by Transcription factor EB (TFEB). TFEB is expressed on lysosomal membranes and translocates to the nucleus, where it induces the transcription of target genes (27). TFEB regulates the expression of multiple genes encoding lysosomal proteins, including both enzymes and membrane proteins. The fact that STG expressing cells had high cytoplasmic TFEB and LAMP2a expression but not nuclear TFEB, suggests that lysosomes are accumulated in the context of reduced lysosomal consumption and inactive lysosomal biogenesis. The increase inLC3B, a membrane marker of autophagosomes (28), also suggests an accumulation of LC3B + autophagosomes and autophagy suppression in STG highly-expressing cancer cells. This is in accordance with a study by Tai et al., showing that oxidative stress-induced senescence is characterized by autophagy impairment and lysosomal dysfunction (29).

Survival analysis showed that STG expression was directly linked with poor prognosis, and this finding was independent of the stage of the disease. Whether this
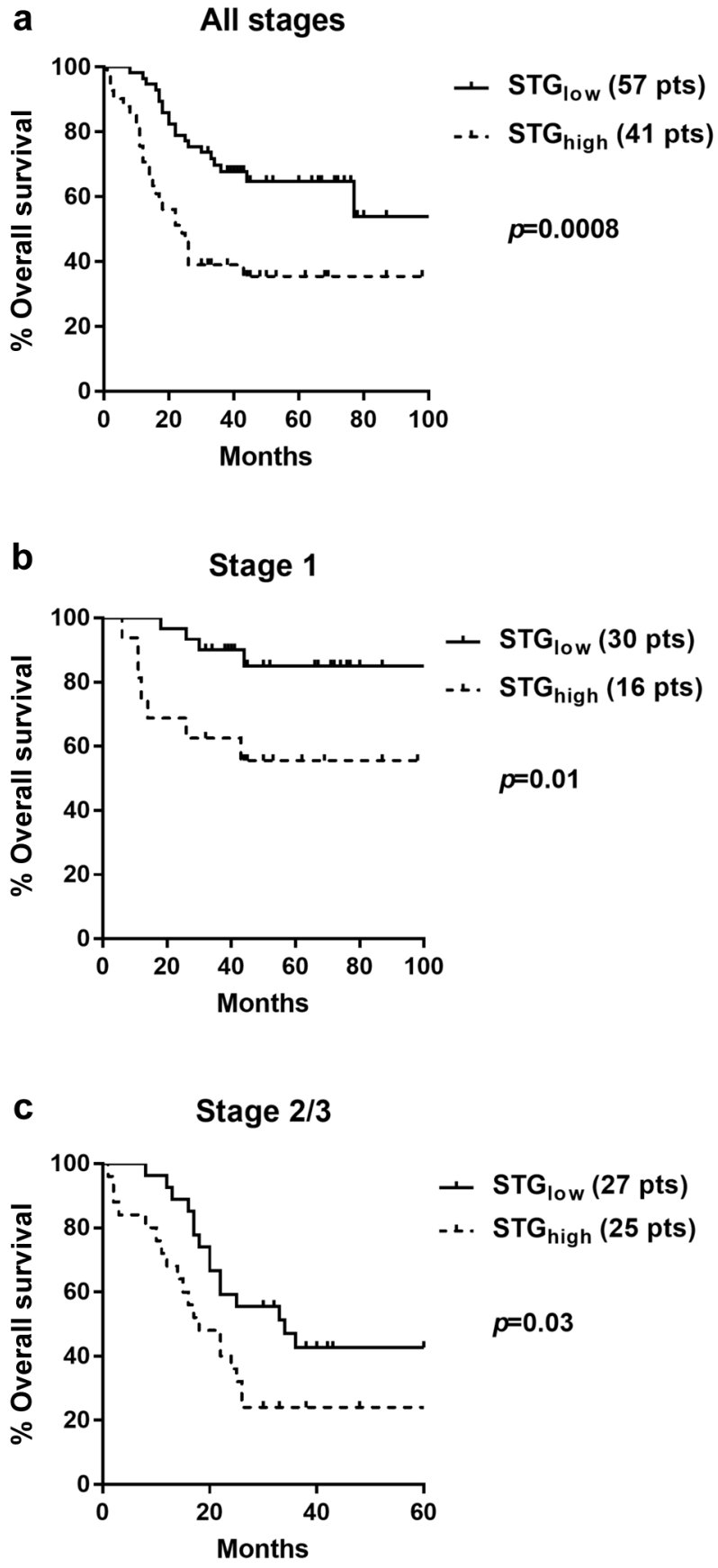

Figure 3. Disease specific Kaplan-Meier overall survival curves: (a) all cases; (b) stage I disease; (c) stage II and III disease.

ominous prognostic relevance of STG expression is related to a direct paracrine effect of senescent cells with a secretory phenotype or an indirect correlation of senescence with tumor aggressiveness related to hypoxia and metabolic pathways demands further investigation. 
It is concluded that SenTraGor ${ }^{\mathrm{TM}}$ provides a reliable methodology to detect lipofuscin accumulation in cancer cells in paraffin-embedded tissues, opening a new field for translational studies focusing on senescence. The direct association of STG expression with hypoxia, glycolysis markers, lysosomal accumulation and autophagy blockage, suggests that STG allows the detection of senescent cells. The abundance of STG/lipofuscin expressing cancer cells was linked with a poor postoperative prognosis of patients with non-small cell lung cancer, bringing forward an eventual role of senescent cells in defining tumor aggressiveness. STG appears as a reliable novel tool for the evaluation of senescence and as a prognostic and predictive tumor feature.

\section{Conflicts of Interest}

There are no conflicts of interest to declare regarding this study.

\section{Authors' Contributions}

AG: Conception and design, analysis and interpretation of data, writing of the paper; MaK: Acquisition of data, immunohistochemistry assessment, writing of the paper; KB: Performed the immunohistochemistry, writing of the paper; MIK: Conception and design, analysis and interpretation of data, writing of the paper.

\section{Acknowledgements}

The study has been financially supported by the Tumour and Angiogenesis Research Group.

\section{References}

1 Gonzalez-Meljem JM, Apps JR, Fraser HC and MartinezBarbera JP: Paracrine roles of cellular senescence in promoting tumourigenesis. Br J Cancer 118: 1283-1288, 2018. PMID: 29670296. DOI: 10.1038/s41416-018-0066-1

2 Lowe SW, Cepero E and Evan G: Intrinsic tumour suppression. Nature 432: 307-315, 2004. PMID: 15549092. DOI: 10.1038/nature 03098

3 Adams PD: Healing and hurting: molecular mechanisms, functions, and pathologies of cellular senescence. Mol Cell 36: 214, 2009. PMID: 19818705. DOI: 10.1016/j.molcel.2009.09.021

4 Demaria M, Ohtani N, Youssef SA, Rodier F, Toussaint W, Mitchell JR, Laberge RM, Vijg J, Van Steeg H, Dollé ME, Hoeijmakers JH, de Bruin A, Hara E and Campisi J: An essential role for senescent cells in optimal wound healing through secretion of PDGF-AA. Dev Cell 31: 722-733, 2014. PMID: 25499914. DOI: 10.1016/j.devcel.2014.11.012

5 Hoenicke L and Zender L: Immune surveillance of senescent cells - biological significance in cancer- and non-cancer pathologies. Carcinogenesis 33: 1123-1126, 2012. PMID: 25499914. DOI: 10.1016/j.devcel.2014.11.012

6 Chen Z, Cao K, Xia Y, Li Y, Hou Y, Wang L, Li L, Chang L and $\mathrm{Li} \mathrm{W}$ : Cellular senescence in ionizing radiation (Review). Oncol
Rep 42: 883-894, 2019. PMID: 31233195. DOI: 10.3892/or.2019. 7209

7 Nguyen HQ, To NH, Zadigue P, Kerbrat S, De La Taille A, Le Gouvello $\mathrm{S}$ and Belkacemi $\mathrm{Y}$ : Ionizing radiation-induced cellular senescence promotes tissue fibrosis after radiotherapy. A review. Crit Rev Oncol Hematol 129: 13-26, 2018. PMID: 30097231. DOI: 10.1016/j.critrevonc.2018.06.012

8 Qin S, Schulte BA and Wang GY: Role of senescence induction in cancer treatment. World J Clin Oncol 9: 180-187, 2018. PMID: 30622926. DOI: 10.5306/wjco.v9.i8.180

9 Hernandez-Segura A, Nehme J and Demaria M: Hallmarks of cellular senescence. Trends Cell Biol 28: 436-453, 2018. PMID: 29477613. DOI: 10.1016/j.tcb.2018.02.001

10 Itahana K, Campisi $\mathrm{J}$ and Dimri GP: Methods to detect biomarkers of cellular senescence: the senescence-associated beta-galactosidase assay. Methods Mol Biol 371: 21-31, 2007. PMID: 17634571. DOI: 10.1007/978-1-59745-361-5_3.

11 Evangelou K and Gorgoulis VG: Sudan Black B, the specific histochemical stain for lipofuscin: a novel method to detect senescent cells. Methods Mol Biol 1534: 111-119, 2017. PMID: 27812872. DOI: $10.1007 / 978-1-4939-6670-7 \_10$

12 Evangelou K, Lougiakis N, Rizou SV, Kotsinas A, Kletsas D, Muñoz-Espín D, Kastrinakis NG, Pouli N, Marakos P, Townsend P, Serrano M, Bartek J and Gorgoulis VG: Robust, universal biomarker assay to detect senescent cells in biological specimens. Aging Cell 16: 192-197, 2017. PMID: 27812872. DOI: $10.1007 / 978-1-4939-6670-7 \_10$

13 Giatromanolaki A, Kouroupi M, Balaska K and Koukourakis MI: Immunohistochemical detection of senescence markers in human sarcomas. Pathol Res Pract 27: 152800, 2019. PMID: 31899047. DOI: 10.1016/j.prp.2019.152800

14 Code E: Research. Code of Practice and Standards. Available at: https://www.hta.gov.uk/sites/default/files/Code\%20E\%20\%20Research\%20Final.pdf [Last Accessed Aug 3, 2020]

15 Giatromanolaki A, Sivridis E, Arelaki S and Koukourakis MI: Expression of enzymes related to glucose metabolism in nonsmall cell lung cancer and prognosis. Exp Lung Res 43: 167-174, 2017. PMID: 28644754. DOI: 10.1080/01902148.2017.1328714

16 Karpathiou G, Sivridis E, Koukourakis MI, Mikroulis D, Bouros D, Froudarakis ME and Giatromanolaki A: Light-chain 3A autophagic activity and prognostic significance in non-small cell lung carcinomas. Chest 140: 127-134, 2011. PMID: 21148243. DOI: $10.1378 /$ chest.10-1831

17 Giatromanolaki A, Kalamida D, Sivridis E, Karagounis IV, Gatter KC, Harris AL and Koukourakis MI: Increased expression of transcription factor EB (TFEB) is associated with autophagy, migratory phenotype and poor prognosis in non-small cell lung cancer. Lung Cancer 90: 98-105, 2015. PMID: 26264650. DOI: 10.1016/j.lungcan.2015.07.008

18 Terman A and Brunk UT: Lipofuscin. Int J Biochem Cell Biol 36: 1400-1404, 2004. PMID: 15147719. DOI: 10.1016/ j.biocel.2003.08.009

19 Kikugawa K, Kato T, Beppu M and Hayasaka A: Fluorescent and cross-linked proteins formed by free radical and aldehyde species generated during lipid oxidation. Adv Exp Med Biol 266: 345-356, 1989. PMID: 2486162. DOI: 10.1007/978-1-4899-5339-1_25

20 Mahmoodi M, Zhang S, Salim S, Hou JS and Garcia FU: Lipofuscin pigment can be used as a prognostic marker in prostatic adenocarcinoma. Ann Diagn Pathol 10: 257-262, 2006. PMID: 16979516. DOI: 10.1016/j.anndiagpath.2005.12.007 
21 Schito L and Semenza GL: Hypoxia-inducible factors: master regulators of cancer progression. Trends Cancer 2: 758-770, 2016. PMID: 28741521. DOI: 10.1016/j.trecan.2016.10.016

22 Alique M, Sánchez-López E, Bodega G, Giannarelli C, Carracedo J and Ramírez R: Hypoxia-inducible factor- $1 \alpha$ : the master regulator of endothelial cell senescence in vascular aging. Cells 9(1), 2020. PMID: 31941032. DOI: 10.3390/cells9010195

23 Carabelli J, Burgueño AL, Rosselli MS, Gianotti TF, Lago NR, Pirola CJ and Sookoian S: High fat diet-induced liver steatosis promotes an increase in liver mitochondrial biogenesis in response to hypoxia. J Cell Mol Med 15: 1329-1338, 2011. PMID: 20629985. DOI: 10.1111/j.1582-4934.2010.01128.x

24 Dillon LM, Williams SL, Hida A, Peacock JD, Prolla TA, Lincoln $\mathrm{J}$ and Moraes CT: Increased mitochondrial biogenesis in muscle improves aging phenotypes in the mtDNA mutator mouse. Hum Mol Genet 21: 2288-2297, 2012. PMID: 22357654. DOI: $10.1093 / \mathrm{hmg} / \mathrm{dds} 049$

25 Yuan Y, Cruzat VF, Newsholme P, Cheng J, Chen Y and Lu Y: Regulation of SIRT1 in aging: Roles in mitochondrial function and biogenesis. Mech Ageing Dev 155: 10-21, 2016. PMID: 26923269. DOI: 10.1016/j.mad.2016.02.003

26 Lee BY, Han JA, Im JS, Morrone A, Johung K, Goodwin EC, Kleijer WJ and DiMaio D and Hwang ES: Senescence associated beta-galactosidase is lysosomal beta-galactosidase. Aging Cell 5: 187-195, 2006. PMID: 16626397. DOI: 10.1111/j.1474-9726. 2006.00199.x
27 Settembre C, Di Malta C, Polito VA, Garcia Arencibia M, Vetrini F, Erdin S, Erdin SU, Huynh T, Medina D, Colella P, Sardiello M, Rubinsztein DC and Ballabio A: TFEB links autophagy to lysosomal biogenesis. Science 332: 1429-1433, 2011. PMID: 21617040. DOI: 10.1126/science.1204592

28 Koukourakis MI, Kalamida D, Giatromanolaki A, Zois CE, Sivridis E, Pouliliou S, Mitrakas A, Gatter KC and Harris AL: Autophagosome proteins LC3A, LC3B and LC3C have distinct subcellular distribution kinetics and expression in cancer cell lines. PLoS One 10: e0137675, 2015. PMID: 26378792. DOI: 10.1371/journal.pone.0137675

29 Tai H, Wang Z, Gong H, Han X, Zhou J, Wang X, Wei X, Ding Y, Huang N, Qin J, Zhang J, Wang S, Gao F, ChrzanowskaLightowlers ZM, Xiang R and Xiao H: Autophagy impairment with lysosomal and mitochondrial dysfunction is an important characteristic of oxidative stress-induced senescence. Autophagy 13: 99-113, 2017. PMID: 27791464. DOI: 10.1080/15548627. 2016.1247143
Received July 17, 2020

Revised August 3, 2020

Accepted August 7, 2020 